\title{
Pneumatic positioning control system using constrained model predictive controller: Experimental repeatability test
}

\author{
Siti Fatimah Sulaiman', M. F. Rahmat ${ }^{2}$, Ahmad Athif Faudzi ${ }^{3}$, Khairuddin Osman ${ }^{4}$, S. I. Samsudin ${ }^{5}$, \\ A. F. Z. Abidin ${ }^{6}$, Noor Asyikin Sulaiman ${ }^{7}$ \\ ${ }^{1,4,5,7}$ Centre for Telecommunication Research and Innovation (CeTRI), Faculty of Electronics and Computer Engineering, \\ Universiti Teknikal Malaysia Melaka, Malaysia \\ ${ }^{2,3}$ School of Electrical Engineering, Universiti Teknologi Malaysia, Malaysia \\ ${ }^{2,3}$ Centre for Artificial Intelligence and Robotics (CAIRO), Universiti Teknologi Malaysia, Malaysia \\ ${ }^{6}$ Faculty of Electrical and Electronics Engineering Technology, Universiti Teknikal Malaysia Melaka, Malaysia
}

\section{Article Info}

Article history:

Received Oct 16, 2020

Revised Mar 2, 2021

Accepted Mar 23, 2021

\section{Keywords:}

Constraint

MPC

Pneumatic system

Position control

System identification

\begin{abstract}
Most of the controllers that were proposed to control the pneumatic positioning system did not consider the limitations or constraints of the system in their algorithms. Non-compliance with the prescribed system constraints may damage the pneumatic components and adversely affect its positioning accuracy, especially when the system is controlled in real-time environment. Model predictive controller (MPC) is one of the predictive controllers that is able to consider the constraint of the system in its algorithm. Therefore, constrained MPC (CMPC) was proposed in this study to improve the accuracy of pneumatic positioning system while considering the constraints of the system. The mathematical model of pneumatic system was determined by system identification technique and the control signal to the valves were considered as the constraints of the pneumatic system when developing the controller. In order to verify the accuracy and reliability of CMPC, repetitive experiments on the CMPC strategy was implemented. The existing predictive controller, that was used to control the pneumatic system such as predictive functional control (PFC), was also compared. The experimental results revealed that CMPC effectively improved the position accuracy of the pneumatic system compared to PFC strategy. However, CMPC not capable to provide a fast response as PFC.
\end{abstract}

This is an open access article under the CC BY-SA license.

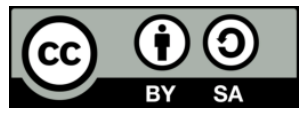

Corresponding Author:

Siti Fatimah Sulaiman

Centre for Telecommunication Research and Innovation (CeTRI)

Faculty of Electronics and Computer Engineering

Universiti Teknikal Malaysia Melaka

76100 Durian Tunggal, Melaka, Malaysia

Email: sitifatimahsulaiman@utem.edu.my

\section{INTRODUCTION}

Essentially, modern pneumatic system incorporates pneumatic cylinder actuator, microprocessor, valves, and various microsensors in a single system; thus, making it more complicated and sophisticated compared to the conventional pneumatic system. However, the complexities of the modern pneumatic system makes the modelling and controlling, especially to acquire an accurate position control of the pneumatic system very challenging due to the issues of parameter uncertainties and nonlinearities [1]. For wider applications, the pneumatic system must have the capability to attain fast response and accurate positioning control [2]. 
Addressing the limitations or constraints of the system in the design of controller for the actual applications of the system is another important aspect to be concerned [3]. This is because, the noncompliance with the prescribed constraints of the system may cause damage to the system and its components, apart from adversely affecting the performance of the control system itself. Proportionalintegral-derivative (PID) [1], [4], sliding mode control (SMC) [5], [6], adaptive [7], [8], fuzzy [9], [10], neural network [11], [12], and predictive [13]-[15] were various control strategies reportedly proposed to control the pneumatic positioning system. Prior studies [14], [15] demonstrated the appropriateness of the predictive controller to control the positioning of the pneumatic system. For the case of the adopted pneumatic system in this study, the review [16]-[18] also revealed the appropriateness of predictive controller as a control strategy for the positioning of the system, given its high speed response with minimal overshoot or even without overshoot in certain cases. Accordingly, Mustafa et al. [16] proposed the generalized predictive controller (GPC) based on bat algorithm (GPC-BA) to control the positioning of the pneumatic system. In 2015, Osman et al. [17] proposed the easy and simple predictive functional controller (PFC) to control the pneumatic system. According to Osman et al., the PFC is capable to provide accurate control with high speed response, robustness, and stability. Three years later, the reduced-order type observer was reportedly applied in the PFC (PFC-ROO) to control the positioning of the pneumatic system [18]. Azira et al. [18] claimed that PFC-ROO provides good response in terms of speed and overshoot compared to the previous PFC which was developed by Osman et al. in [17]. Nonetheless, these studies [17], [18] did not consider the constraints of the pneumatic system in their control algorithms. As previously described, the non-compliance with the prescribed constraints may damage the pneumatic system and its components and adversely affect the overall performance of the control system itself. Hence, this study regarded the significance of considering the constraints during the development of the controller.

This study proposed the model predictive controller (MPC) with the constraints on the control signal of the pneumatic valves as the control strategy for the positioning of the pneumatic system used. The MPC was chosen in this study as it has the capability to handle constraint [19], [20]. In particular, the added constraints in MPC prevent the wind-up phenomenon [21]. Additionally, MPC has the capability to predict the future output of the process [19], control variety of processes (from a relatively simple process to a more complex process), handle different levels of the process control structure [20], easily deal with multivariable processes [20], and handle nonlinear processes (which is frequently found in the industries) [20]. In this study, the control signal to the input valves were considered as the constraints of the pneumatic system used, since it affects the system response (i.e. the position of the pneumatic cylinder stroke). The main aim of the proposed controller is to provide the pneumatic positioning system with simultaneously accurate, fast, and at the same time respect the constraints of the system. In order to verify the accuracy and reliability of the CMPC, repetitive experiments on the control strategy were implemented. Subsequently, the performance of the CMPC was analysed and compared with the recent control (PFC) method that were tested on the same plant.

This paper is divided into 4 sections. The background of the study is discussed in section 1 . The modelling process of the pneumatic system used in this study and the procedures in designing a CMPC to perform the control task are explained in section 2. The experimental results using CMPC and PFC were presented and discussed in section 3, and the overall findings of the study are concluded in section 4.

\section{RESEARCH METHOD}

\subsection{Modelling of pneumatic system using system identification technique}

Figure 1 presents the real pneumatic system used in this study, and its main components. The pneumatic system in this study was equipped with five main components, which were integrated into a single actuator. These components included: i) Optical encoder, ii) Stripe code on a guide rod, iii) Pressure sensor, iv) Valves, and programmable system on chip (PSoC) microcontroller board. The operations of pneumatic positioning system based on experimental setup are schematically shown in Figure 2.

The optical encoder in Figure 2 was used to detect cylinder rod position by reading the constructed stripes code on the guide rod, while the pressure sensor was used to read the pressure inside chamber 2 of the cylinder. The readings of both pressure sensor and encoder were subsequently sent to the analog-to-digital converter (ADC) and counter module of PSoC, respectively, for feedback as well as to decide the position of the cylinder stroke. The pneumatic system used in this study was mainly controlled by only one chamber whereas the other chamber was fixed at constant pressurised air. Regulating the air pressure in chamber 2 between $0 \mathrm{MPa}$ and $0.6 \mathrm{MPa}$ controlled the left and right movements of the actuator and provided pressurised air at constant pressure $(0.6 \mathrm{MPa})$ in chamber 1 . In this regard, two valves were used to control chamber 2. As illustrated in Figure 2, two miniature valves, which were attached at the end of the pneumatic cylinder, were used to control the air inlet and air outlet of the cylinder. More specifically, valve 1 controlled the air 
inlet, while valve 2 controlled the air outlet (exhaust). Both valves were operated at $24 \mathrm{~V}$ in order to control the pneumatic stroke movement. Overvoltage to the valves may cause damage to the pneumatic system and its components. For this study, the extension (right movement) and retraction (left movement) of the cylinder stroke were manipulated by the duty cycle of pulse-width modulator (PWM) signal to drive the valves in regulating the pressure in chamber 2 . Therefore, the control signal, $u$ (from the controller) was initially converted into PWM signal for each valve in order to operate these valves.

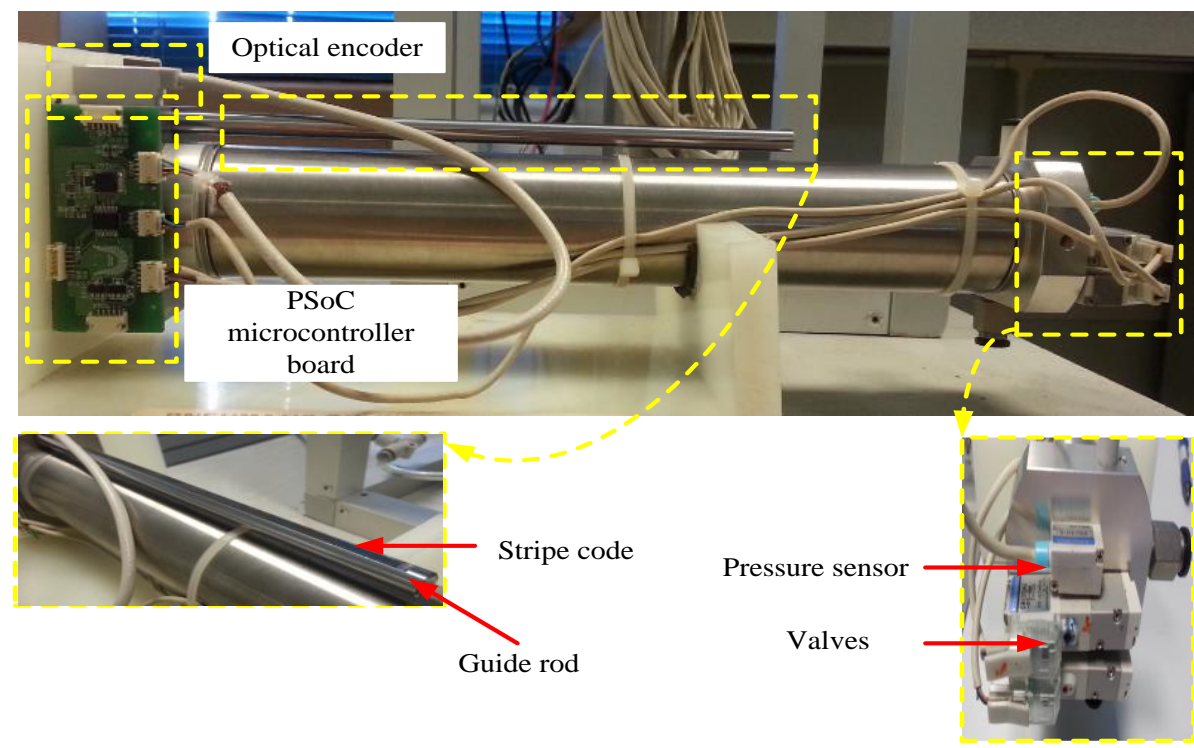

Figure 1. Main components of the pneumatic system

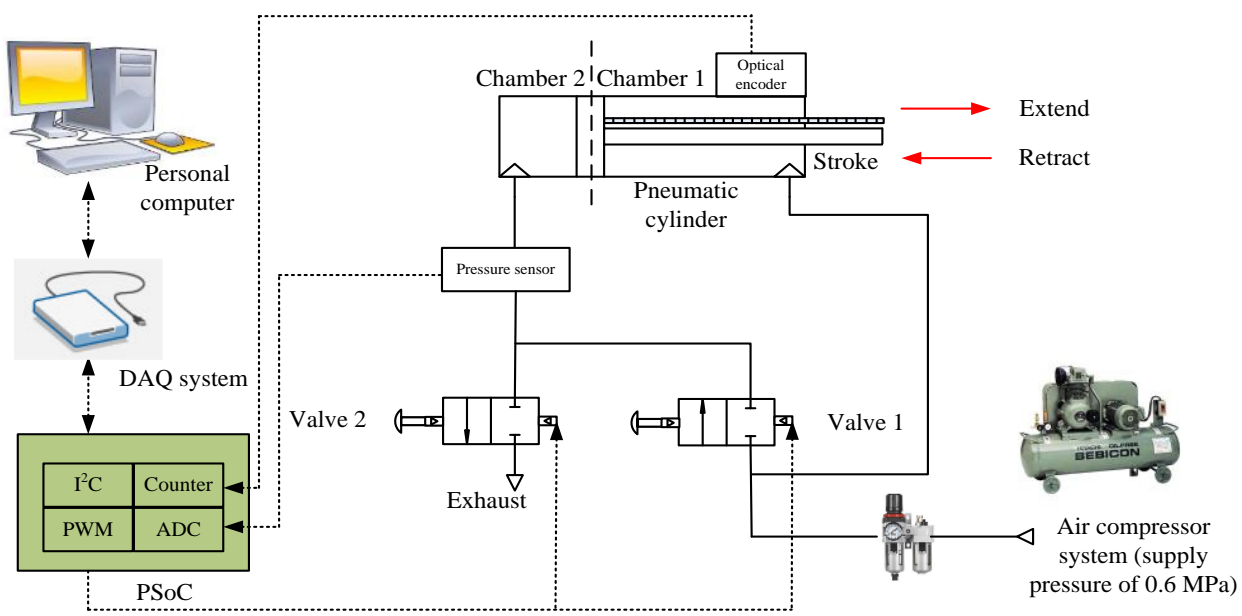

Figure 2. Schematic diagram of pneumatic positioning system

System identification technique was applied in this study to acquire real-time model that represents the dynamics of the pneumatic system used. To model the pneumatic system using system identification technique, an experimental design and data collection was performed [22], [23], so that the data of input and output from real-time experiment can be collected. In this study, an open-loop experimental test for input and output data collection was conducted 3 times in order to repeat data extraction as well as to produce consistent performances of the input and output response for reliability of the data. The experiments were conducted within $20 \mathrm{~s}$ with the sampling time $\left(T_{S}\right)$ of $10 \mathrm{~ms}$. Figure 3 shows the plot of data of measured input and output acquired from real-time experiment. The input signal as shown in Figure 3(a) was injected as excitation signal and the output of this system as shown in Figure 3(b) was recorded. It should be noted 
here that, the input signal in Figure 3(a) was repeated 3 times, and the output signal in Figure 3(b) was actually the recorded average values of the three measured output responses.

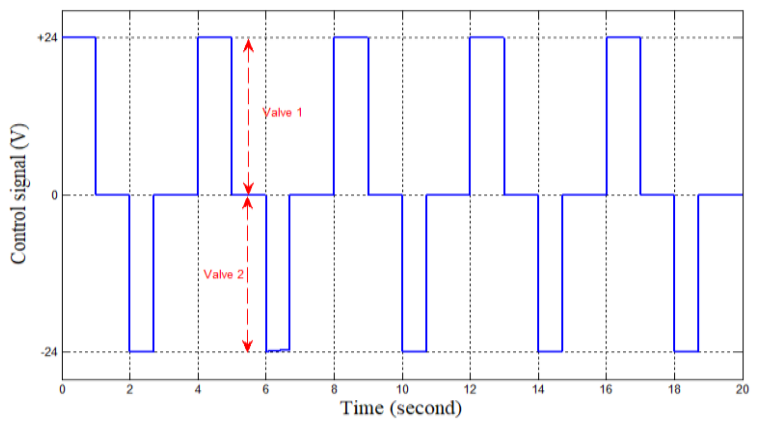

(a)

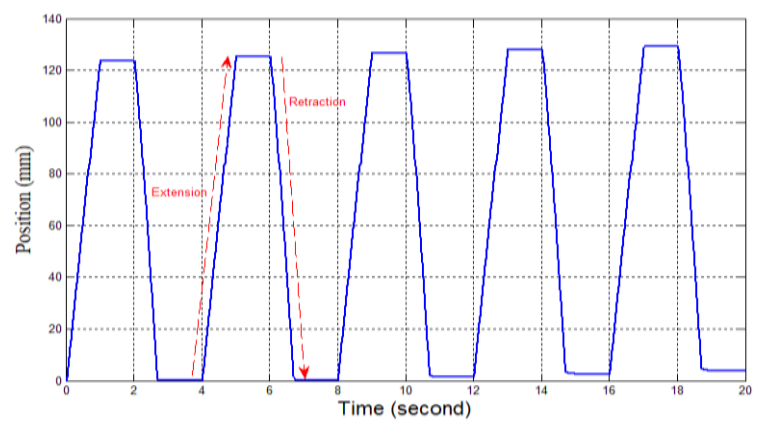

(b)

Figure 3. Plot of measured data: (a) input and (b) output

In this study, 2,000 measurements of input and output data were collected from open-loop test; the input data consisted of continuous step input signal applied to the on/off valves of the system, while the output data was the measured position of cylinder stroke. For modelling using system identification technique, the data of input and output were divided into two sets; one set for training (estimation) and the other set for the validation of the identified model. Figure 4 illustrates the estimation and validation process carried out using MATLAB system identification tool.

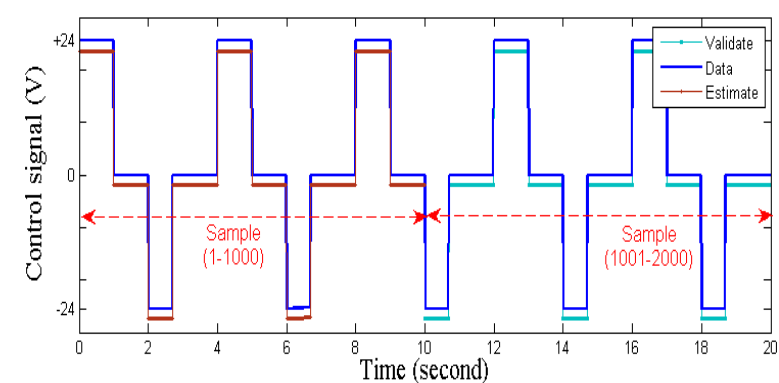

(a)

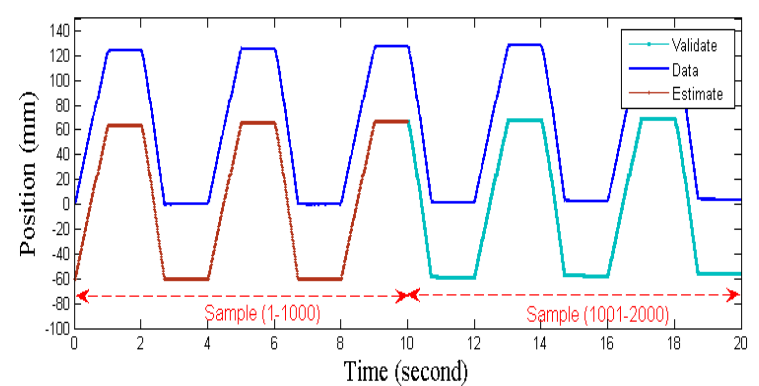

(b)

Figure 4. Estimation and validation process of measured data using MATLAB system identification tool,

(a) Input and (b) Output

Based on the plots as illustrated in Figure 4, 50\% of the first data set contained 1,000 data of input and output (sample 1-1000) collected from real-time experiment were used for model training (estimation), meanwhile the remaining 50\% of the second data sets (sample 1001-2000) was used for testing (validation). In this study, an auto-regressive with eXogenous input (ARX) with third-order system was selected as the model structure of pneumatic system. ARX model has a simple structure, make it capable to provide relatively faster computation and estimation. As the pneumatic system used in this study is complex, therefore, the use of complex model structure is not recommended as it will make the closed-loop system of the pneumatic more complex. The use of higher-order models (i.e. fourth-order and fifth-order) for ARX were not considered in this study since it does not always warrant higher accuracy of the system. Also, increasing the complexity of the model typically increases the uncertainties in the parameter estimation. In (1) expresses the discrete-time state-space of the pneumatic system dynamics based on third-order ARX model structure, which was estimated using MATLAB system identification tool.

$$
A_{p}=\left[\begin{array}{ccc}
0 & 1 & 0 \\
0 & 0 & 1 \\
0.1284 & -0.9976 & 1.8690
\end{array}\right], B_{p}=\left[\begin{array}{l}
0 \\
0 \\
1
\end{array}\right], C_{p}=\left[\begin{array}{lll}
0.0016 & 0 & 0
\end{array}\right], D_{p}=[0]
$$


Where $A_{p}, B_{p}, C_{p}$, and $D_{p}$ are the pneumatic system matrices with dimension, $n_{p}=3$. The identified thirdorder ARX model successfully provides a good output fitting (91.11\% of fitting) to the measured output obtained from the real-time experiments. In terms of stability, the identified model is also stable since it successfully provides all the system's poles inside the unit circle $(0.1887,0.6811$, and 0.9992$)$. Therefore, modelling the pneumatic system based third-order linear ARX model is sufficient to represent the real pneumatic system utilised in this study.

\subsection{Controller design}

\subsubsection{Constrained model predictive controller (CMPC)}

This study proposed model predictive controller (MPC) as the controller to control the pneumatic positioning system. For this study, MPC uses the identified process model which was obtained using system identification technique to predict the future output (position of cylinder stroke). The control strategy in this study is to ensure and maintain the position of pneumatic cylinder stroke at a desired position regardless of the disturbances that were introduced into the system. This study assumed single-input single-output (SISO) system for the pneumatic system in a state-space model. Considering that the third-order discrete state-space model (as previously described in (1)) as the plant model, the representation of augmented model of the pneumatic positioning system utilised for this study was as in (1):

$$
A=\left[\begin{array}{cccc}
0 & 1 & 0 & 0 \\
0 & 0 & 1 & 0 \\
0.1284 & -0.9976 & 1.8690 & 0 \\
0 & 0.0016 & 0 & 1
\end{array}\right], B=\left[\begin{array}{l}
0 \\
0 \\
1 \\
0
\end{array}\right], C=\left[\begin{array}{llll}
0 & 0 & 0 & 1
\end{array}\right]
$$

In this study, the control signal to the valves was the manipulated variable whereas the position of the cylinder stroke was the controlled variables. The MPC was used to determine the future adjustments of the control signals to the valves. In order to do so, MPC predicts the future plant outputs and performs the control actions accordingly by solving the optimal future control actions (specifically, cost function and constraints) within a moving time horizon window. The cost function, $J$, with respect to the control objective of this study was expressed in (3):

$$
J=\sum_{m=1}^{N_{p}} x(k+m \mid k)^{T} Q x(k+m \mid k)+\eta^{T} R \eta
$$

where $\eta$ is the optimal solution of parameter vector, $Q$ and $R$ are the weighting matrices with $Q=C^{T} C \geq$ 0 and $R>0$. The cost function in (3) is based on the minimization of the error between the set-point signal and the output signal (desired position of the pneumatic cylinder stroke). The optimal solution of parameter vector, $\eta$ in (3) based on the minimisation of cost function using predictive control algorithms can be expressed as shown in (4).

$$
\eta=-\Omega^{-1} \psi x(k)
$$

where $\Omega=\sum_{m=1}^{N_{p}} \phi(m) Q \phi(m)^{T}+R, \psi=\sum_{m=1}^{N_{p}} \phi(m) Q A^{m}$.

Thus, the control law based on the discrete-time Laguerre function can be realised in (5):

$$
\Delta u(k)=L(0)^{T} \eta
$$

The control law in (5) is represented in the form of linear state feedback control, which is expressed in (6):

$$
\Delta u(k)=-K_{m p c} x(k)(6)
$$

where $K_{m p c}=L(0)^{T} \Omega^{-1} \psi, x(k)=\left[\Delta x_{i p a}(k)^{T} \quad e(k)\right]^{T}$

$e(k)$ denotes the error signal between the predicted output, $y(k)$, and set-point signal, $r(k)$. The closed-loop characteristic equation of the MPC system is expressed as $\operatorname{det}\left(\lambda I-\left(A-B K_{m p c}\right)\right)=0$, in which their closed-loop eigenvalues reveals its stability. The closed-loop system is considered stable if all the eigenvalues of the closed-loop matrix $\left(A-B K_{m p c}\right)$ are inside the unit circle. Hence, this study applied the following MPC law: 


$$
u(k)=\Delta u(k)+u(k-1)
$$

As previously described, this study considers a control signal to the input valves as the constraints of the systems, since it affects the system response (i.e. the position of the cylinder stroke). Thus, this study controlled this signal to ensure that it remains within a certain range allowed by the system. Typically, the system response significantly deteriorates and may generate overshoot when the signal exceeds the maximum allowable value, which is known to occur rather frequently in real-time environment. Considering the specifications and limitations of the hardware used, the maximum amplitude value allowed for the extension and retraction of the cylinder stroke during operation of this study was set to $+24 \mathrm{~V}$ (for valve 1) and $-24 \mathrm{~V}$ (for valve 2), respectively. Thus, the signal from the MPC to the valves was constrained between $+24 \mathrm{~V}$ and $-24 \mathrm{~V}$. Supposed that the minimum and maximum limits on the control signal to the pneumatic valves are $u_{\text {min }}=-24$ and $u_{\max }=+24$, and $u(k)=\sum_{i=0}^{k-1} \Delta u(k)$, the inequality constraints for future time $k$, $k=1,2, \cdots$ are expressed as:

$$
-24 \leq M \eta+u(k-1) \leq+24
$$

where $M=\left[\begin{array}{cccc}\sum_{i=0}^{k-1} L_{1}(i)^{T} & 0_{2}{ }^{T} & \cdots & 0_{m}{ }^{T} \\ 0_{1}{ }^{T} & \sum_{i=0}^{k-1} L_{2}(i)^{T} & \cdots & 0_{m}{ }^{T} \\ \vdots & \vdots & \vdots & \vdots \\ 0_{1}{ }^{T} & 0_{2}{ }^{T} & \cdots & \sum_{i=0}^{k-1} L_{m}(i)^{T}\end{array}\right]$

where $u(k-1)$ is the previous control signal and $0_{k}{ }^{T}$ is a row vector with dimensions as in $L_{k}(0)^{T}$. The control signal with constraints on the input valves in (8) can also be expressed as (9).

$$
u_{\min } \leq u(k) \leq u_{\max }
$$

Table 1 summarizes the values of MPC parameters used in this study. $N_{c}$ and $N_{p}$ in Table 1 act as tuning parameters that affect the prediction of future output and stability of the closed-loop system. $N_{c}$ dictates the number of parameters to capture the future control trajectory, while $N_{p}$ represents the length of optimization window. Generally, the value of $N_{c}$ is chosen to be less than (or equal to) the value of $N_{p}$. $N_{c}$ for this study was set at 3 since it provides $<10 \%$ of $O S$, and $0 \mathrm{~mm}$ of steady-state error $\left(e_{S S}\right)$. The overshoot $(O S)$ of the transient response was also increased with increasing $N_{c}$. The closed-loop system was also unstable when $N_{p}=1$ and $N_{p}=2$ were used to solve the optimization problem. In other words, the pneumatic positioning closed-loop system was unstable and the $O S$ of the transient response was also increased when small value of $N_{p}$ was used. Hence, it can be concluded that the closed-loop predictive control system is not necessarily stable with small $N_{p}$. Thus, in order to guarantee closed-loop stability of the pneumatic positioning system as well as to avoid overshoot and oscillate response of the pneumatic positioning system, $N_{c}$ and $N_{p}$ for this study was set at 3 and $20\left(N_{c} \ll N_{p}\right)$, respectively.

Table 1. Description of the MPC parameters

\begin{tabular}{ccc}
\hline & Control parameters \\
\hline Name of parameter & Abbreviation & Value \\
Prediction horizon & $N_{p}$ & 20 \\
Control horizon & $N_{c}$ & 3 \\
Scaling factor of Laguerre network & $\alpha$ & 0.1 \\
Weighting matrices & $R$ & 0.1 \\
& & {$\left[\begin{array}{llll}0 & 0 & 0 & 0 \\
0 & 0 & 0 & 0 \\
0 & 0 & 0 & 0 \\
0 & 0 & 0 & 1\end{array}\right]$} \\
\hline
\end{tabular}

\subsubsection{Predictive functional controller (PFC)}

PFC uses the same approach as MPC (i.e. prediction of the future outputs, and calculation of the manipulated variables) for an optimal control. In other words, PFC is based on the same principles that are using an internal model, specification of a reference trajectory, and determination of the control law. Similar to MPC design, this study also used state-space form for the purpose of designing PFC. Thus, the discretetime state-space model of the pneumatic system in (1) was used for this purpose. In order to formulate the 
PFC control law, the desired reference trajectory must be first known. In this study, the desired closed-loop dynamic was placed into the desired reference trajectory to ensure the stability of the closed-loop system. Given the actual set-point $(r)$, the first-order lag of the loop set-point $(w)$ reference trajectory can be defined as in (10):

$$
w(k+i \mid k)=r(k)-(r(k)-y(k)) \Psi^{i}
$$

where $y(k)$ is the most recent measured output, and $\Psi$ is a tuning parameter that represents system's closedloop pole. In order to ensure the stability of the closed-loop system of this study, the value of $\Psi$ must be within the following range: $0 \leq \Psi<1$. Previous study by Osman et al. in [17] used 0.95 to represent $\Psi$. Therefore, for the purpose of comparing the performance of the pneumatic positioning system, this study also used the same value, which is 0.95 to represent the value of $\Psi$. PFC is made of coincidence points. When the predicted output and set-point coincide, a coincidence points is formed. Typically, there are only one or two coincidence points. In order to enforce equality of the predictions and the reference trajectory at a number of points, the control law of PFC can be determined by solving the future control moves such in (11).

$$
y(k+n)=w(k+n)
$$

Where $n$ is the number of coincidence points. The focus of this study is only on one coincidence point. Therefore, at a single coincidence point, the future control moves can be described in (12).

$$
y(k+n)=w(k+n)=r(k)-(r(k)-y(k)) \Psi^{i}
$$

The substitution of (10) into (11) provides (13).

$$
y(k+n)=P x_{i p a}(k)+H u(k-1)=r(k)-(r(k)-y(k)) \Psi^{i}
$$

Rewriting (13), the PFC control law at a single coincidence point can be described as:

$$
\begin{aligned}
& u(k)=-H^{-1}\left[P x_{i p a}(k)+\left(r(k)-(r(k)-y(k)) \Psi^{i}\right)\right] \\
& u(k)=-K_{c} x_{i p a}(k)+P_{c} r(k)
\end{aligned}
$$

where

$$
K_{c}=-H^{-1}\left[P-\Psi^{i} y(k)\right] \text { and } P_{c}=-H^{-1}\left[1-\Psi^{i}\right]
$$

\section{RESULTS AND DISCUSSION}

This section presented the performances of the proposed control strategy (CMPC) and PFC strategy in controlling the positioning system of the pneumatic system through experimental work. Figure 5 shows the real-time pneumatic positioning control performances using CMPC and PFC strategies for $50 \mathrm{~mm}, 100 \mathrm{~mm}$, and $150 \mathrm{~mm}$ distances. Repetitive experiments on the control strategies were implemented in order to perform a statistical analysis of the experimental results. The experiments were repeated in order to verify the accuracy and reliability of the controllers. In this study, each experiment conducted on each control strategy (CMPC and PFC) was repeated 10 times to determine the capabilities of each controller, especially the proposed control strategy to perform repeating experiments and producing consistent performances. In this analysis, several values of distance $(50 \mathrm{~mm}, 100 \mathrm{~mm}$, and $150 \mathrm{~mm})$ were provided for comparison purposes, and the step signal was applied as the input signal. Each test was conducted for $20 \mathrm{~s}$ without any load attached to the end of the cylinder stroke.

Table 2 to Table 4 provide statistical analysis for each distance using CMPC and PFC control strategies, which were repeated 10 times. In these tables, the performances of the system's transient (i.e. rise time $\left(t_{r}\right)$, settling time $\left(t_{s}\right)$, overshoot $(O S)$, and steady-state error $\left(e_{s s}\right)$ ) between CMPC and PFC were compared. From the data provided in Table 2 to Table 4, the responses of $O S$ revealed that PFC provided zero standard deviation and zero standard error, for all the positioning distances. Therefore, indicates that PFC gives exactly the mean value of $O S$ for all distances, which means that every single experiment performed for each distance also resulted in a system response without $O S$. For the analysis of $e_{s S}$, CMPC strategy was capable of providing the lowest readings of standard deviation and standard error compared to 
PFC, which signifies the consistency and reliability of CMPC in providing consistent position accuracy compared to PFC.

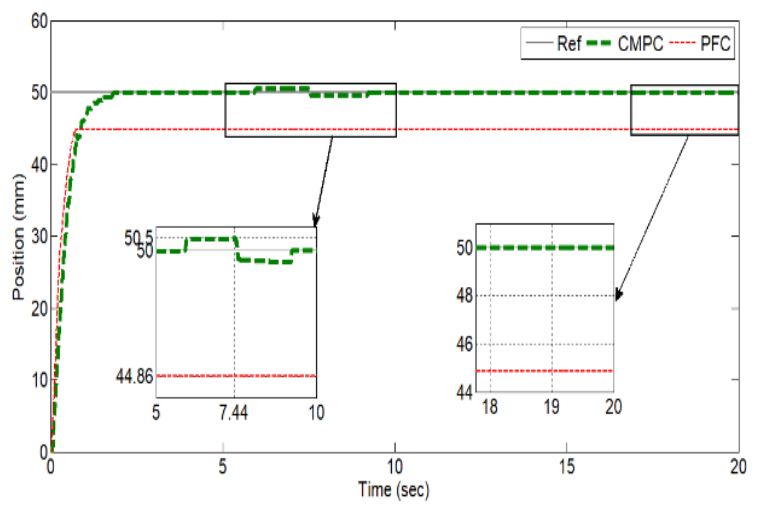

(a)

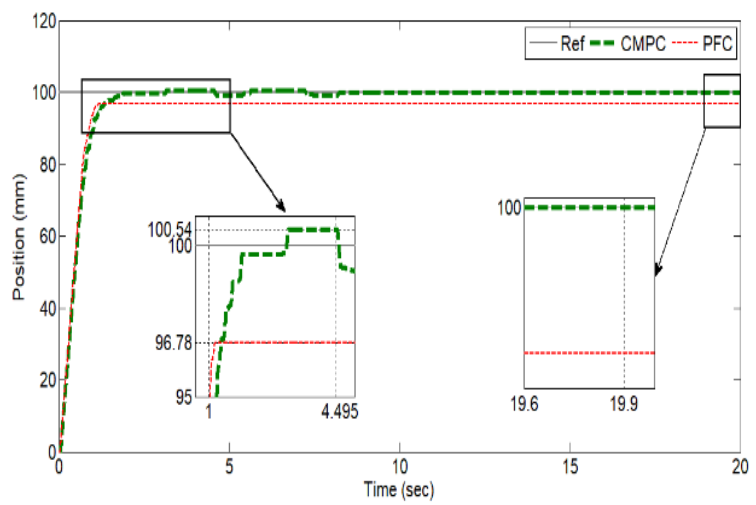

(b)

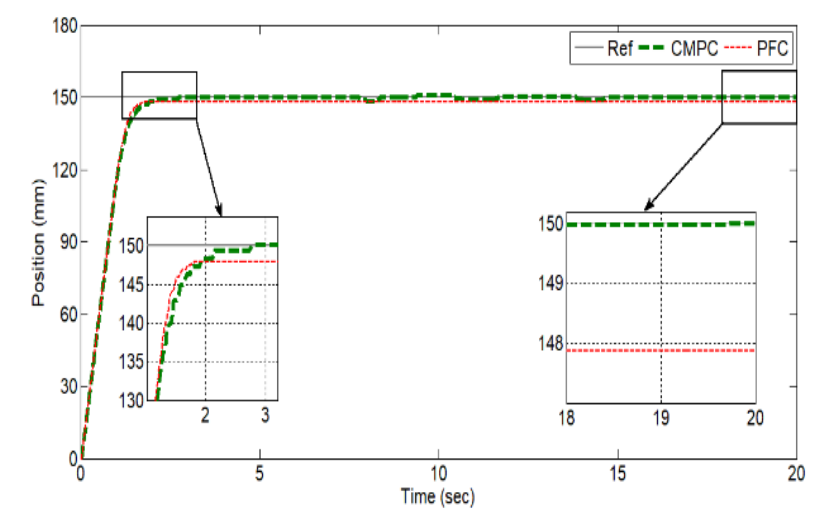

(c)

Figure 5. Real-time pneumatic positioning control performances using CMPC and PFC strategies when position distance at: (a) $50 \mathrm{~mm}$, (b) $100 \mathrm{~mm}$, and (c) $150 \mathrm{~mm}$

Table 2. Statistical analysis of transient response performance for pneumatic positioning system

\begin{tabular}{cccccc}
\multicolumn{6}{c}{ at $50 \mathrm{~mm}$ of distance } \\
\hline Control strategies & Criterion & $t_{r}(s)$ & $t_{s}(s)$ & OS $(\%)$ & $e_{s s}(\mathrm{~mm})$ \\
\hline \multirow{4}{*}{ CMPC } & Mean & 0.790 & 1.470 & 0.692 & 0.096 \\
& Variance & 0.001 & 0.010 & 0.217 & 0.010 \\
& Standard deviation & 0.035 & 0.101 & 0.465 & 0.098 \\
& Standard error & 0.011 & 0.032 & 0.147 & 0.031 \\
PFC & Mean & 0.462 & 0.665 & 0 & 5.309 \\
& Variance & 0 & 0 & 0 & 0.016 \\
& Standard deviation & 0.006 & 0.013 & 0 & 0.128 \\
& Standard error & 0.002 & 0.004 & 0 & 0.040 \\
\hline
\end{tabular}

Table 3. Statistical analysis of transient response performance for pneumatic positioning system

\begin{tabular}{cccccc}
\multicolumn{6}{c}{ at $100 \mathrm{~mm}$ of distance } \\
\hline Control strategies & Criterion & $t_{r}(s)$ & $t_{s}(s)$ & $O S(\%)$ & $e_{s s}(\mathrm{~mm})$ \\
\hline \multirow{4}{*}{ CMPC } & Mean & 0.878 & 1.545 & 0.536 & 0.029 \\
& Variance & 0 & 0.002 & 0.027 & 0.001 \\
& Standard deviation & 0.013 & 0.049 & 0.164 & 0.026 \\
& Standard error & 0.004 & 0.015 & 0.052 & 0.008 \\
PFC & Mean & 0.714 & 1.043 & 0 & 3.629 \\
& Variance & 0 & 0.001 & 0 & 0.066 \\
& Standard deviation & 0.009 & 0.038 & 0 & 0.258 \\
& Standard error & 0.003 & 0.012 & 0 & 0.082 \\
\hline
\end{tabular}


Table 4. Statistical analysis of transient response performance for pneumatic positioning system at $150 \mathrm{~mm}$ of distance

\begin{tabular}{cccccc}
\hline Control strategies & Criterion & $t_{r}(s)$ & $t_{s}(s)$ & $O S(\%)$ & $e_{s s}(\mathrm{~mm})$ \\
\hline \multirow{4}{*}{ CMPC } & Mean & 1.107 & 1.772 & 0.479 & 0.035 \\
& Variance & 0 & 0.001 & 0.040 & 0.001 \\
& Standard deviation & 0.006 & 0.023 & 0.200 & 0.025 \\
& Standard error & 0.002 & 0.007 & 0.063 & 0.008 \\
& Mean & 1.053 & 1.618 & 0 & 1.973 \\
& Variance & 0 & 0.073 & 0 & 0.382 \\
& Standard deviation & 0.010 & 0.270 & 0 & 0.618 \\
& Standard error & 0.003 & 0.085 & 0 & 0.196 \\
\hline
\end{tabular}

Table 5 summarizes the mean value of the transient response performance obtained from the conducted repeatable experiments, meanwhile Figure 6 illustrates every single change that occurred in the transient response performance. It can be seen that the proposed CMPC was capable of controlling the pneumatic positioning system. The comparison with the previous control strategy (PFC) shows that the pneumatic system positioning system controlled by the proposed CMPC strategy was unable to control the system as fast as PFC. Clearly, the inclusion of constraints on the control signal to the pneumatic valves in the MPC algorithm caused the speed response $\left(t_{r}\right.$ and $\left.t_{s}\right)$ of the pneumatic positioning system to be very slow and less aggressive, compared to PFC. The findings were consistent with the studies by [19], [20], which demonstrated that the inclusion of constraints in the MPC algorithm slowed down the response time in the system output since it required more computational burden to optimize the cost function (compared to the unconstrained MPC).

Table 5. Summary of the mean value of transient response performance obtained from repeated experiments

\begin{tabular}{llccll}
\hline Distance $(\mathrm{mm})$ & Control strategy & $t_{r}(s)$ & $t_{s}(s)$ & $0 S(\%)$ & $e_{s s}(\mathrm{~mm})$ \\
\hline \multirow{2}{*}{50} & CMPC & 0.790 & 1.470 & 0.692 & 0.096 \\
& PFC & 0.462 & 0.665 & 0 & 5.309 \\
\multirow{2}{*}{100} & CMPC & 0.878 & 1.545 & 0.536 & 0.029 \\
& PFC & 0.714 & 1.043 & 0 & 3.629 \\
150 & CMPC & 1.107 & 1.772 & 0.479 & 0.035 \\
& PFC & 1.053 & 1.618 & 0 & 1.973 \\
\hline
\end{tabular}

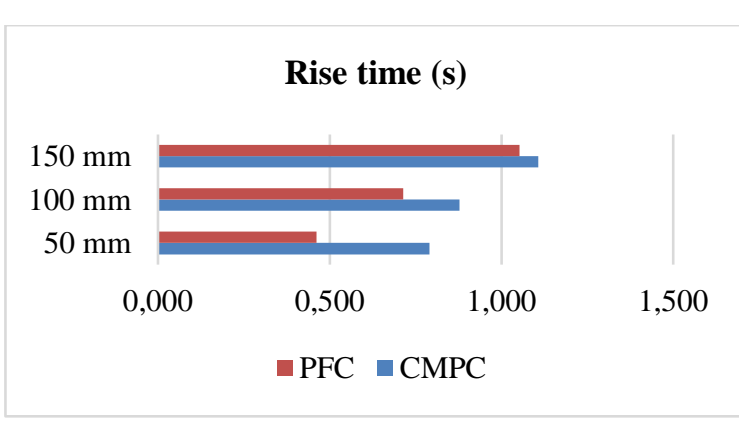

(a)

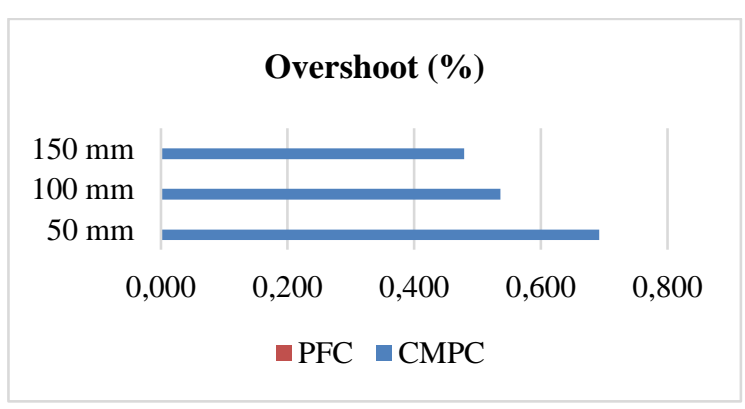

(c)

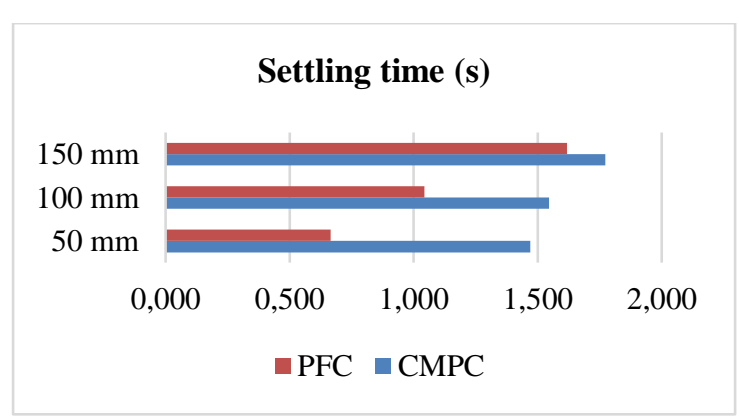

(b)

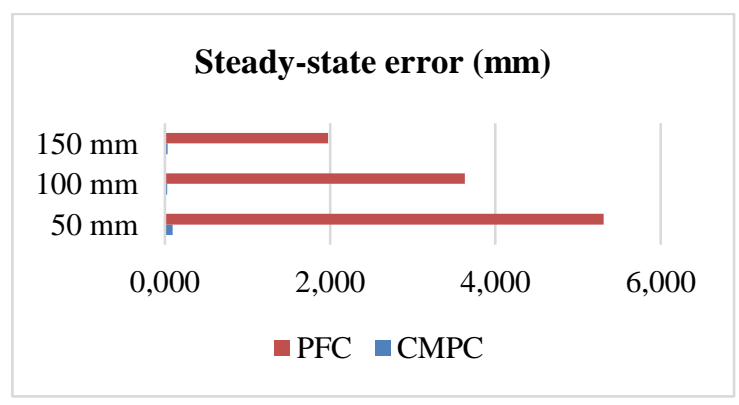

(d)

Figure 6. Analysis of the transient response of repeated experiments: (a) rise time $\left(t_{r}\right)$, (b) settling time $\left(t_{s}\right)$, (c) overshoot $(O S)$, and $(\mathrm{d})$ steady-state error $\left(e_{s S}\right)$ 
The analysis of overshoot $(O S)$ also revealed that PFC consistently provided $0 \%$ of $O S$, for all the positioning distances. Considering that the overshoot in the transient response of the pneumatic system was mainly due to the compressibility of air [11], the maximum allowable overshoot for this study was set to 10 $\%$ as similarly used in other studies [24], [25]. Although there was a slight overshoot $O S$ at the beginning of the positioning response (for all positioning distances) using CMPC; however, it is considered acceptable to control the pneumatic positioning system since it manages to provide $<10 \%$ of overshoot for all positioning distances.

As presented in Table 5 and Figure 6, a significant difference that must be highlighted between PFC and CMPC is regarding the steady-state error $\left(e_{s s}\right)$ performance. Furthermore, it is also worthy of noting, that PFC failed to give a good positioning accuracy as CMPC. PFC indicated $>1.973 \mathrm{~mm}$ error for all position distances, while the proposed CMPC consistently produced a $<0.029 \mathrm{~mm}$ error for all position distances. Furthermore, the proposed CMPC can implement constraints in the pneumatic system and hence, making it the preferred choice to be considered in any system with constraints.

\section{CONCLUSION}

A control strategy namely constrained model predictive controller (CMPC) was proposed to control the position of pneumatic system used in this study. The aim of the proposed controller is to consider the constraints of the pneumatic system and improves the transient response of the pneumatic positioning control system. In this study, the control signal to the pneumatic valve was considered as the constraints of the pneumatic system when developing the controller. This study primarily focused to evaluate the capability of the proposed controller as a practical strategy to control the position of pneumatic system and to improve the transient response performance of the system. In order to verify the accuracy, reliability, and consistent performances of the proposed control strategy, the positioning control were repeated 10 times. The performance of the CMPC was analysed and compared with the recent control method (PFC) that were tested on the same plant. From the experimental results of this study, it was confirmed that the proposed CMPC was successfully implemented in real-time environment and satisfies constraints of the pneumatic system used. Also, the CMPC was proven to be capable in improving the steady-state accuracy; thus, providing higher accuracy in the position control of the pneumatic cylinder stroke. Even though the CMPC was able to provide accurate positioning control of the system, however, CMPC is unable to give fast response of the positioning system as PFC did. For wider applications, pneumatic system must have the capability to attain fast speed response with high accuracy. Therefore, for future work, this study considered that the speed response of the system can be improved through certain modifications in the CMPC algorithm.

\section{ACKNOWLEDGEMENTS}

The authors would like to acknowledge Universiti Teknikal Malaysia Melaka (UTeM) under Short Term Research Grant Scheme (PJP/2019/FKEKK(1C)/S01657) and Ministry of Higher Education (MOHE) of Malaysia for their support.

\section{REFERENCES}

[1] R. B. van Varseveld and G. M. Bone, "Accurate position control of a pneumatic actuator using on/off solenoid valves," in IEEE/ASME Transactions on Mechatronics, vol. 2, no. 3, pp. 195-204, Sep. 1997, doi: $10.1109 / 3516.622972$.

[2] D. Saravanakumar, B. Mohan and T. Muthuramalingam, "A Review On Recent Research Trends in Servo Pneumatic Positioning Systems," Precision Engineering, vol. 49, pp. 481-492, 2017, doi: 10.1016/j.precisioneng.2017.01.014.

[3] J. Deng, V. Becerra, and R. Stobart, "Input Constraints Handling in an MPC/Feedback Linearization Scheme," International Journal of Applied Mathematics and Computer Science, vol. 19, pp. 219-232, 2009, doi: 10.2478/v10006-009-0018-2.

[4] J. Wang, J. Pu and P. Moore, "A Practical Control Strategy for Servo-Pneumatic Actuator Systems," Control Engineering Practice, vol. 7, no. 12, pp. 1483-1488, 1999, doi: 10.1016/S0967-0661(99)00115-X.

[5] A. K. Paul, J. E. Mishra and M. G. Radke, "Reduced order sliding mode control for pneumatic actuator," in IEEE Transactions on Control Systems Technology, vol. 2, no. 3, pp. 271-276, Sep. 1994, doi: 10.1109/87.317984.

[6] S. R. Pandian, Y. Hayakawa, Y. Kanazawa, Y. Kamoyama and S. Kawamura, "Practical Design of a Sliding Mode Controller for Pneumatic Actuators," Journal of Dynamic Systems, Measurement, and Control, vol. 119, no. 4, pp. 666-674, 1997, doi: 10.1115/1.2802376.

[7] J. E. Bobrow and F. Jabbari, "Adaptive Pneumatic Force Actuation and Position Control," Journal of Dynamic Systems, Measurement, and Control, vol. 113, no. 2, pp. 267-272, 1991, doi: 10.1115/1.2896374. 
[8] G. A. Medrano-Cerda, C. J. Bowler and D. G. Caldwell, "Adaptive position control of antagonistic pneumatic muscle actuators," Proceedings 1995 IEEE/RSJ International Conference on Intelligent Robots and Systems. Human Robot Interaction and Cooperative Robots, Pittsburgh, PA, USA, 1995, pp. 378-383 vol. 1, doi: 10.1109/IROS.1995.525824.

[9] S. Chillari, S. Guccione and G. Muscato, "An experimental comparison between several pneumatic position control methods," Proceedings of the 40th IEEE Conference on Decision and Control (Cat. No.01CH37228), Orlando, FL, USA, vol. 2, 2001, pp. 1168-1173, doi: 10.1109/CDC.2001.981043.

[10] H. Schulte and H. Hahn, "Fuzzy State Feedback Gain Scheduling Control of Servo Pneumatic Actuators," Control Engineering Practice, vol. 12, no. 5, pp. 639-650, 2004, doi: 10.1016/S0967-0661(03)00148-5.

[11] H. K. Lee, G. S. Choi, and G. H. Choi, "A study on Tracking Position Control of Pneumatic Actuators," Mechatronics, vol. 12, no. 6, pp. 813-831, 2002, doi: 10.1016/S0957-4158(01)00024-1.

[12] T. D. C. Thanh and K. K. Ahn, "Nonlinear PID Control to Improve the Control Performance of 2 Axes Pneumatic Artificial Muscle Manipulator Using Neural Network," Mechatronics, vol. 16, no. 9, pp. 577-587, 2006, doi: 10.1016/j.mechatronics.2006.03.011.

[13] G. Andrikopoulos, G. Nikolakopoulos, and S. Manesis, "Pneumatic Artificial Muscles: A Switching Model Predictive Control Approach," Control Engineering Practice, vol. 21, no. 12, pp. 1653-1664, 2013, doi: 10.1016/j.conengprac.2013.09.003.

[14] L. Zhang and X. Zhuan, "Optimal Operation of Heavy-Haul Trains Equipped With Electronically Controlled Pneumatic Brake Systems Using Model Predictive Control Methodology," in IEEE Transactions on Control Systems Technology, vol. 22, no. 1, pp. 13-22, Jan. 2014, doi: 10.1109/TCST.2013.2238235.

[15] G. M. Bone, M. Xue, and J. Flett, "Position Control of Hybrid Pneumatic-Electric Actuators Using Discrete-Valued Model-Predictive Control," Mechatronics, vol. 25, pp. 1-10, 2015, doi: 10.1016/j.mechatronics.2014.10.009.

[16] N. D. Mustafa, A. A. M. Faudzi, A. F. Z. Abidin, K. Osman and K. Suzumori, "Generalized predictive controller using Bat algorithm for double acting pneumatic cylinder," 2013 IEEE Student Conference on Research and Developement, Putrajaya, Malaysia, 2013, pp. 572-576, doi: 10.1109/SCOReD.2013.7002657.

[17] K. Osman, A. A. M. Faudzi, M. F. Rahmat, O. F. Hikmat, and K. Suzumori, "Predictive Functional Control with Observer (PFC-O) Design and Loading Effects Performance for a Pneumatic System," Arabian Journal for Science and Engineering, vol. 40, pp. 633-643, 2015, doi: 10.1007/s13369-014-1421-z.

[18] A. R. Azira, K. Osman, S. I. Samsudin, S. F. Sulaiman, "Predictive Functional Controller (PFC) with Novel Observer Method for Pneumatic Positioning System," Journal of Telecommunication, Electronic and Computer Engineering (JTEC), vol. 10, no. 2-6, pp. 119-124, 2018.

[19] E. F. Camacho and C. Bordons, "Model predictive control," Advanced textbooks in control and signal processing, Springer-Verlag, 2004.

[20] L. Wang, "Model predictive control system design and implementation using MATLAB®," Springer Science \& Business Media, 2009.

[21] J. M. Maciejowski, "Predictive control with constraints," Pearson Education, 2000.

[22] S. F. Sulaiman, M. F. Rahmat, A. A. M. Faudzi and K. Osman, "Design of unconstrained and constrained model predictive control for pneumatic actuator system: Set-point tracking," 2015 IEEE Conference on Systems, Process and Control (ICSPC), Bandar Sunway, Malaysia, 2015, pp. 112-117, doi: 10.1109/SPC.2015.7473569.

[23] S. F. Sulaiman, M. F. Rahmat, A. A. M. Faudz, and K. Osman, "Enhanced Position Control for Pneumatic System by Applying Constraints in MPC Algorithm," International Journal of Electrical and Computer Engineering (IJECE), vol. 7, no. 3, pp. 1633-1642, 2017, doi: 10.11591/ijece.v7i3.pp1633-1642.

[24] M. Heidari and H. Homaei, "Improving the Pneumatic Control Valve Performance using a PID Controller," Turkish Journal of Engineering and Environmental Sciences, vol. 38, pp. 240-247, 2015,doi: 10.3906/muh-1301-8.

[25] M. H. Beni, H. Homaei, E. Kiani, and F. Abbaspour, "Dynamic Modeling and Simulation of Control System for Pneumatic Servo Valve Using Piezoelectric Actuator," Ciência e Natura, vol. 37, pp. 153-161, 2015, doi: $10.5902 / 2179460 X 20842$. 